\title{
DO RANDOM VELOCITY FIELDS INHIBIT OR INDUCE GRAVITATIONAL COLLAPSE AND FRAGMENTATION ?
}

\author{
PHILIPPE CHANTRY, ROLAND GRAPPIN, JACQUES LÉORAT \\ Observatoire de Meudon, DAEC, 92190 Meudon - France \\ E-mail CHANTRY@FRMEU51.BITNET
}

Recent numerical works on self-gravitating turbulence (Bonazzola et al., 1987; Léorat, Passot, Pouquet 1990) tend to show that turbulent velocity fields could act as a "turbulent" pressure, i.e. inhibit the collapse of self-gravitating clouds. This effect is efficient when the caracteristic scale of the turbulent flow is small compared to the Jeans's length. When these two scales are comparable, we show (Chantry, Grappin, Léorat, 1990) that the turbulence do no more inhibit the collapse, but seems to trigger it.

We performed two- and three-dimensional simulations of self-gravitating isothermal fluids, using a pseudo-spectral method. In the three-dimensional simulations, turbulence is sustained by a large-scale external shear force, so that a statistically stationary state is reached before gravitational term is put on. With $32^{3}$ resolution, we create such stationary states with Mach numbers around 1 , Reynolds numbers around 40 and with less than $10 \%$ of compressible kinetic energy. When self-gravity is added, we observe that

a) when the system is initially linearly unstable (following Jeans's criterion), the turbulence accelerates the gravitational collapse;

b) when the system is initialy linearly stable (or marginally stable), the turbulence trigger the collapse of scales near but smaller than the Jeans's scale.

In both cases, we are able to follow numerically the gravitational collapse up to density contrasts ( dens $_{\max } /$ dens $_{\min }$ ) between a few hundreds and $10^{6}$.

We also simulated two-dimensional decaying flows with very simple initial conditions :

$$
U_{x}=-U \cos x \sin y ; U_{y}=U \sin x \cos y ; \text { density }=1 .
$$

These simulations show that a purely incompressible flow is able to generate very quickly density fluctuations at the hyperbolic stagnation points of the flow (saddle points). When self-gravity is present and the system is Jeans unstable, this nonlinear growth of the density is a starting point for a further phase of gravitational growth which leads to collapse (Sasao, 1973). When the system is gravitationally 
stable and isothermal, the behaviour of the fluid depends on the initial Mach number. If the Mach number is low, the condensations decay after a few turnover times (Fig. 1a); when it is high enough, the condensations become frozen and the system reaches a quasi-stationary state (Fig 1 b) with high density contrast (greater than $300)$. When the system is gravitationally stable and the polytropic index is smaller than unity, the quasi-stationary state may evolve into a further collapsing phase (Fig 1c) leading to density contrasts greater than 5000.

A simple analysis predicts that if one compresses a given Jeans stable mass of polytropic gas in a space of dimension $\mathrm{D}$, the mass may become Jeans unstable after the compression if the polytropic index $\gamma$ of the gas is below a limit depending on D. If $\mathrm{D}=2$, this limit is $\gamma=1$ and if $\mathrm{D}=3$, it is $4 / 3$. This could explain the previous results. In all the cases, the initial growth of the resulting condensed objects is due to non-linear compression at the caracteristic scale of the flow, so that turbulence seems to be an effective mechanism for the fragmentation of the clouds. This effect, which favors collapse at the integral scale of the turbulent flow could act together with the "turbulent pressure" effect, which inhibits collapse at scales much larger than that of the flow.

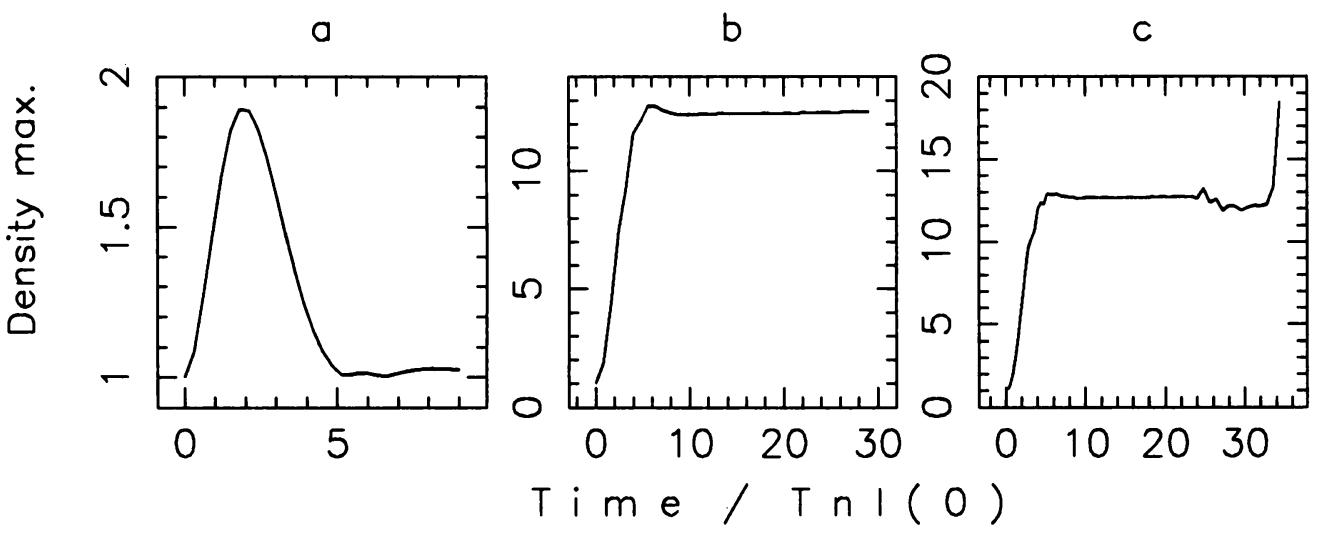

Fig. 1. Time evolution of the maximum density reached in two-dimensional simulations with initial conditions (1). a) initial rms Mach number $\bar{M}_{0}=0.35$; polytropic index $\gamma=$ 1 ; b) $\bar{M}_{0}=0.94 ; \gamma=1$; c) $\bar{M}_{0}=0.94 ; \gamma=0.9$

\section{References}

Bonazzola, S., Falgarone, E., Heyvaerts, J., Pérault, M. and Puget, J.-L. : 1987, "Jeans collapse in a turbulent medium." Astron. Astrophys., 172, 293-298

Chantry, P., Grappin, R. and Léorat, J. : 1990, in preparation.

Léorat, J., Passot, T. and Pouquet, A. : 1990, "Influence of supersonic turbulence on self-gravitating flows." Mon. Not. R. astr. Soc., 243, 293-311

Sasao, T. : 1973, "On the generation of density fluctuation due to turbulence in selfgravitating media." Publ. Astron. Soc. Japan , 25, 1-33 